Inmaculada Montero García ${ }^{1}$

Teresa Baena Reina ${ }^{2}$

Universidad de Granada (España)
Doctora en Pedagogía, Universidad de Granada (España); imontero@ugr.es

2 Maestra en Educación Especial y Licenciada en Pedagogía por la Universidad

de Granada (España); terelus@correo.ugr.es

\section{Experiencias innovadoras en el ámbito de la educación infantil en España. El valor de las relaciones intergeneracionales}

\section{Resumen}

La educación infantil es, en la actualidad, una oportunidad de desarrollo de capacidades para la integración de los niños y niñas en el mundo que les rodea. En este proceso, las personas mayores tienen mucho que aportar, puesto que son una fuente de experiencias y conocimientos de la que se puede nutrir toda la sociedad. Más adelante exponemos los nuevos roles y percepciones de los niños hacia las personas mayores como base de la intergeneracionalidad, mientras que, para finalizar el artículo, se presenta la experiencia de OFECUM como asociación intergeneracional.

Palabras clave: Relaciones intergeneracionales, Educación Infantil, personas mayores, experiencias.

\section{Innovative Experiences in the field of Primary Education in Spain. Value of intergenerational relationships}

\section{Summary}

Primary education is, nowadays, an opportunity for development of capabilities for integration of children in the world around them. In this process, older people have much to contribute, since they're a source of experience and knowledge that can feed all the society. Later we expose the new roles and perceptions of children to the elderly as intergenerationality base, and ends the article with OFECUM experience as intergenerational association.

\section{Keywords: Intergenerational} Relationships, Primary

Education, Elder people, Experiences.

\section{Experiências inovadoras no domínio do ensino primário na Espanha. Valor das relações intergeracionais}

\section{Resumo}

A educação infantil é, na actualidade, uma oportunidade de desenvolvimento de capacidades para a integração dos meninos e das meninas no mundo a volta deles. Neste processo, as pessoas maiores têm muito para dar, porque elas sao uma fonte de experiências y de conhecimentos das quais se pode alimentar toda a sociedade. Mais a frente vamos mostrar os novos papeis e percepção dos meninos até as pessoas maiores com base a intergeracionalidade e acabar o articulo com a experiência da OFECUM como associação de intergeracionalidade.

Palavras-chave: Relações Intergeracionais, Educação Infantil, Pessoas Maiores, Experiências. 


\section{Fines y principios de la educación infantil en España}

$\mathrm{H}^{\circ}$ oy en día, la comprensión de la educación infantil se ha modificado sustancialmente. Lejos de entender esta etapa como un período sin una clara finalidad en sí mismo, en la actualidad se orienta a que niños y niñas desarrollen al máximo sus capacidades, procurando que adquieran aprendizajes que les permitan interpretar gradualmente el mundo que les rodea y actúen en él.

Según el último informe publicado por UNICEF (Fondo Internacional de Emergencia de las Naciones Unidas para la Infancia) en España (2012), la tasa neta de escolaridad en el primer ciclo de Educación Infantil (0, 1 y 2 años) subió al 26 '5\% para el año 2010, en comparación con 2009, que fue de un $24,6 \%$. Parece evidente que, al menos desde la educación reglada, su representatividad y el valor concedido por instancias públicas y la propia familia, es cada vez mayor.

$\mathrm{Su}$ desarrollo comprende aprendizajes relevantes, significativos y motivadores para los niños. Así mismo, y dado el carácter cambiante de nuestra sociedad, la escolaridad busca integrar de forma transversal el desarrollo de valores democráticos, cívicos y éticos, la diversidad cultural, la sostenibilidad, la cultura de la paz, los hábitos de consumo y vida saludable y la utilización del tiempo de ocio; así como contribuir a la superación de las desigualdades por razón de género cuando las hubiere, el respeto de los Derechos Humanos y de las libertades fundamentales y los valores que preparan a las niñas y niños para asumir una vida responsable en una sociedad libre y democrática ${ }^{3}$.

Por su parte, las propuestas pedagógicas y actividades educativas en los centros de educación infantil han de respetar las características propias del crecimiento y del aprendizaje. Consecuentemente, los maestros y maestras y demás profesionales de la educación infantil, han de atender dichas características, partir de los conocimientos previos, necesidades y motivaciones de cada uno de ellos, propiciar la participación activa, fo-

Para complementar, se recomienda revisar la ORDEN de 5 de agosto de 2008, por la que se desarrolla el Currículo correspondiente a la Educación Infantil en Andalucía. BOJA, núm. 169, 26 de agosto de 2008, pág. 17. mentar sus aportaciones, estimular el desarrollo de sus potencialidades y facilitar su interacción con personas adultas, con los iguales y con el medio.

Del mismo modo, el equipo educativo tiene como una de sus funciones prioritarias la planificación de las relaciones con las familias de los niños y niñas, propiciando la comunicación e implicación de las madres y padres en la vida de la escuela, a fin de que éstos coparticipen en los procesos de aprendizaje de sus hijos. Los profesionales de la educación y las familias habrán de colaborar en la educación y la crianza de los niños y niñas, así, su relación ha de basarse en la corresponsabilidad, porque lo que le ocurre a los niños acaba, más pronto o más tarde, repercutiendo inevitablemente en todos nosotros (UNICEF, 2012).

Para ello, la educación infantil se fundamenta en el conocimiento del contexto familiar y la facilitación de los cauces de una mutua colaboración que contemplen el respeto a la diversidad. Al tiempo, los profesionales han de mantener una relación permanente con las familias de los niños y niñas, facilitando situaciones y modos de comunicación y colaboración, promoviendo su presencia y participación en la vida de los centros.

Es por ello que, desde los centros educativos, se trabajan en este sentido acciones formativas orientadas al apoyo y la formación de las familias en materia educativa, entre las que se llevan a cabo cursos monográficos, talleres, grupos de reflexión y sesiones de intercambios de experiencias, a fin de que la familia y la escuela se conviertan en comunidades de prácticas compartidas.

En este contexto, incluimos la experiencia que vamos a exponer. Para nosotras, conocer y difundir este trabajo supone, en gran medida, una forma de concebir y comprender el carácter diverso y heterogéneo de la sociedad de la que todos formamos parte. Creemos firmemente que, en el marco de un mundo tantas veces segregador y poco colaborativo, es preciso y pertinente desarrollar procesos de aprendizaje mutuo, donde ya desde las primeras etapas se persiga el desarrollo de ciudadanos más capaces, reflexivos, implicados y comprometidos con la comunidad a la que pertenecen.

Por su parte, el trabajo con las personas de más edad nos ha hecho apreciar que ellas no sólo tienen mucho que aportar, 
sino que en sí mismas constituyen un modelo de experiencia y conocimiento a través de distintas formas de diálogo y comunicación que, también con los más pequeños, está teniendo una repercusión importante en el ámbito educativo.

La sociedad tiene la responsabilidad compartida de contribuir al desarrollo de las extraordinarias potencialidades de los niños y niñas en estas primeras edades, que se produce como resultado del aprendizaje y la socialización. La escuela desarrolla en esta estructura diferentes funciones, entre otras, las de proporcionar una infancia de múltiples relaciones y oportunidades. Por este camino facilitará la construcción de la propia identidad y potenciará y estimulará diferentes modos de relación e identidad, en términos de pluralidad, de los distintos grupos en el reconocimiento de las diferencias y multiplicidad, también con las personas de más edad.

La escuela se convierte así en un lugar de vidas y relaciones compartidas entre numerosas personas adultas y niños y niñas. En este contexto, la comunicación es clave para el aprendizaje infantil, proporcionando posibilidades diversas de intercambio de experiencias a través de múltiples formas de interacción, expresión y representación, y facilita así un sentimiento de pertenencia, la adopción de posiciones diferentes, la capacidad de ver sus propias experiencias a la luz de los demás, debatir y defender las elecciones propias, abordar situaciones nuevas, regular su propia acción. Resulta conveniente, pues, practicar una pedagogía de la escucha y el diálogo.
Antes de exponer las experiencias llevadas a cabo en tal sentido, a fin de comprender mejor el contexto que estamos considerando, vamos a exponer brevemente cuál es la significación y la fundamentación de las relaciones intergeneracionales, así como las percepciones de los niños y niñas hacia las personas de más edad en la actualidad de la sociedad española.

\section{Nuevos roles y percepciones de los niños y niñas hacia las personas mayores en la sociedad actual. El valor de las relaciones intergeneracionales}

Como su propio nombre indica, cuando aludimos a la expresión "relaciones intergeneracionales", hablamos de las relaciones que se establecen entre generaciones, es decir, entre personas que comparten un mismo período etario. Ello significa que muchas de sus vivencias, percepciones e intereses, pueden ser similares por haber coexistido en un tiempo socio-histórico concreto (Montero, 2003).

Esta potencialidad, de llegar a ser "uno mismo", es algo que va desarrollándose a lo largo de toda la existencia, "desde la cuna a la tumba", de manera que el hombre no es un ser acabado, sino en constante realización personal. Todo ello desemboca en la necesidad, no sólo de "co-existir", sino de "con-vivir"

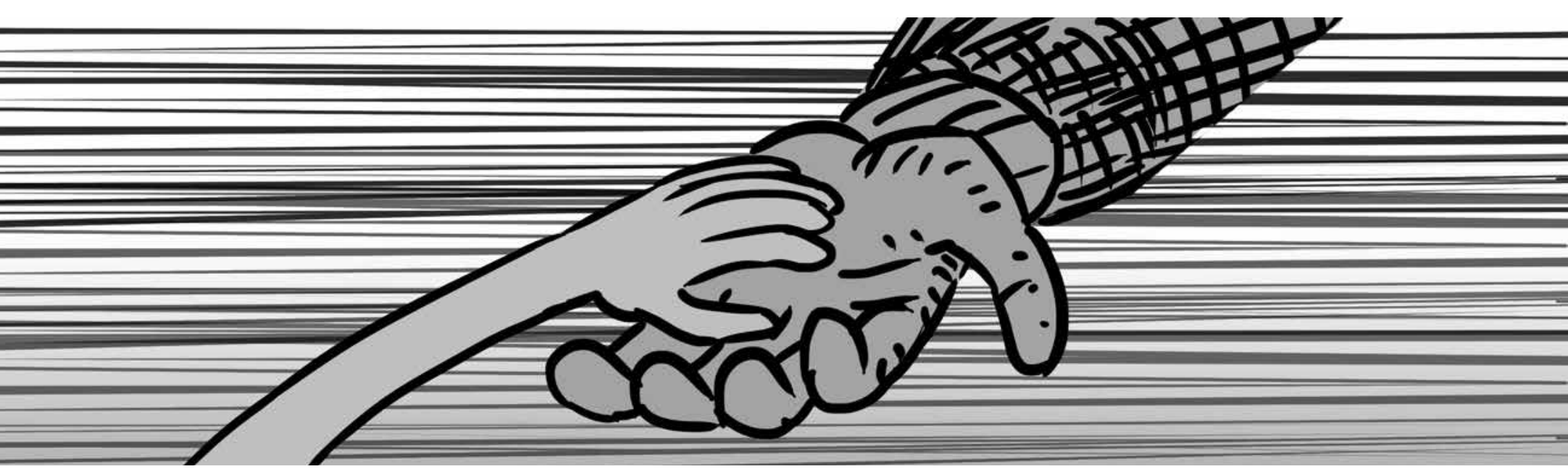

96 Educación y Ciudad No24 Enero - Junio de 2013 ISSN 0123-0425 Pp. 93-105 


\section{Sin ánimo de entrar en juicios de valor acerca de la bondad o no de estos espacios, lo cierto es que cada cual, en función de la edad, tiene determinados lugares donde desarrollar su formación"}

con nuestros semejantes. Las relaciones intergeneracionales no son más que una consecuencia natural de tal existencia, son algo tan antiguo y tan valioso como la misma humanidad. Otra cosa son los cambios históricos y socio-económicos que han favorecido, y a veces casi diríamos, impuesto, que exista una "segregación" por edades en la cotidianeidad social, laboral y, por supuesto, desde el mismo campo educativo.

Hoy, los más pequeños van a guarderías o jardines de infancia, y los que son un poco mayores al colegio donde, desde la educación infantil (3-6 años), se los "divide" en función de la edad. Los jóvenes, por su parte, han de pasar igualmente por instituciones formales que imparten sus enseñanzas en niveles reglados y dentro de los espacios determinados a tal fin. Las personas mayores tienen también "su sitio", ya sea en los centros de día, residencias, centros de estancia diurna, aulas de mayores...

Sin ánimo de entrar en juicios de valor acerca de la bondad o no de estos espacios, lo cierto es que cada cual, en función de la edad, tiene determinados lugares donde desarrollar su formación, su trabajo o su divertimento. Raros son los ámbitos de encuentro comunes a diversas generaciones. Desde nuestra experiencia, provocar momentos de diálogo y comunicación intergeneracional, y proponer procesos donde tengan cabida el desarrollo de valores y la reflexión acerca de lo trabajado, es un ejercicio altamente positivo tanto para los niños y niñas participantes, como para el resto de adultos que colaboran en este intercambio (Montero y Bedmar, 2009; Montero, García Mínguez y Bedmar, 2011). Evidentemente, resulta difícil argüir en contra del hecho de que:

"La cooperación humana está vinculada a tendencias particularmente exacerbadas que se expresan espontáneamente y corresponden a lo que vienen a ser fuertes y sofisticadas predisposiciones "pro-sociales", que incluyen preferencias que contemplan a otros, colaboración y comportamientos impulsados por una preocupación por el

\section{bienestar de otros" (Rochat, 2012, p. 9).}

Lo cierto es que la sociedad no puede considerarse completa ni puede realmente evolucionar, si sus grupos de población no se interrelacionan y, aún más, si no se conocen. La coeducación entre generaciones permite vivir de manera más real los unos con los otros.

Según datos del IMSERSO 4 (2009) en su informe Percepción en niños y adolescentes de las personas mayores, la mayor longevidad y, sobre todo, la mayor claridad con que se abordan los procesos de envejecimiento por parte de las personas mayores ${ }^{5}$, hacen que los niños y niñas se impliquen más en las relaciones con este grupo de población.

Existe una nueva forma de trato con los abuelos y abuelas (y por extensión con los mayores), que conlleva responsabilizarse mutuamente de algunas de las actividades comunes en el contexto familiar o de la familia extensa (que no convive pero está cerca): acudir al médico, a las actividades extraescolares, o "salir" se convierte en el inicio de una tendencia que sitúa a las personas mayores en un plano de más "igualdad" o cercanía con los menores.

Las personas mayores aportan entonces su experiencia y lo que ellos mismos están buscando en una edad que se ofrece más dilatada y más rica en recursos. Esto hace que exista en los propios niños una percepción de la longevidad ligada a la actividad general de las personas. Los niños perciben como rasgo definitorio inicial la mayor capacidad de estar dispuestos que tienen los abuelos, lo cual supone no sólo que se encuen-

4 IMSERSO: Instituto de Mayores y Servicios Sociales en España. El IMSERSO es la Entidad Gestora de la Seguridad Social para la gestión de los Servicios Sociales complementarios de las prestaciones del Sistema de Seguridad Social, y en materia de personas mayores y personas en situación de dependencia.

5 En España utilizamos la expresión "personas mayores" en equiparación a lo que, en América Latina se denomina "adultos mayores". 


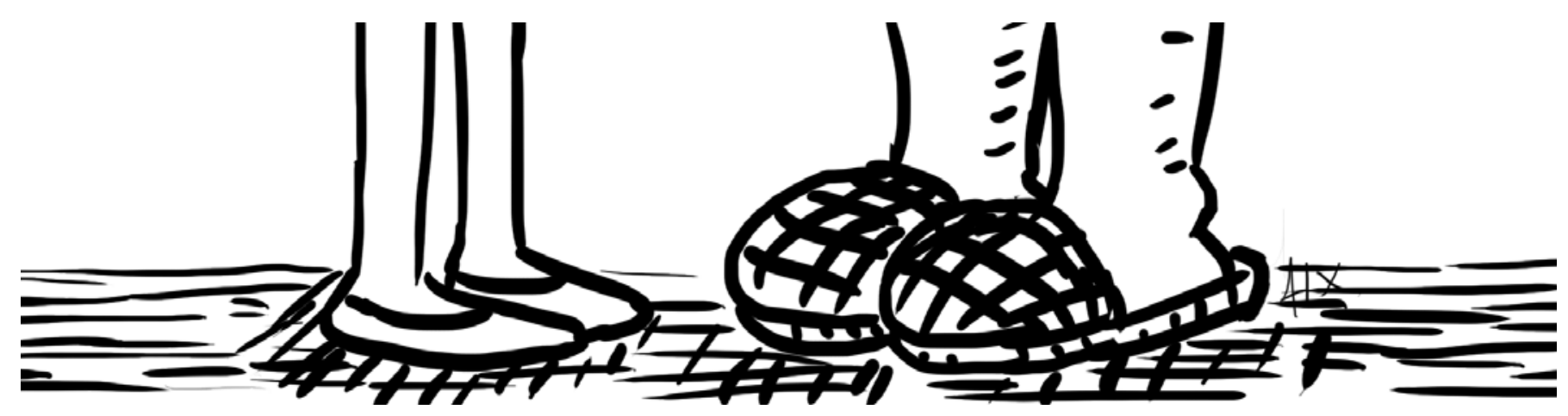

tran mejor ellos, sino que pueden tener mejor relación con los menores y con toda la familia.

Así, la familia en su sentido más amplio pasa a ser un ámbito privilegiado, donde coexisten con mayor intensidad las dificultades y también las ventajas de las relaciones intergeneracionales. Pero tampoco escapa del dinamismo social. Los abuelos en muchas ocasiones ya no viven con el resto de la familia como antes, donde llegaban a cohabitar cuatro generaciones bajo un mismo techo. Las cada vez más amplias ocupaciones educativas y laborales, y la incursión activa de la mujer en el mercado de trabajo, han llevado en cierto modo a destinar a la persona mayor a lugares específicos de encuentro con personas de su misma edad, ya sea de forma transitoria o permanente.

Según la profesora Bazo (1990 y 1999), en realidad la familia actual sólo es formalmente nuclear. Ha pasado a ser una "familia extensa modificada", de modo que distintas familias nucleares viven unidas por lazos emocionales y afectivos. También la atención que desde siempre se ha dedicado a padres y madres ancianos, se ha transformado en una nueva responsabilidad filial de características peculiares.

Por otro lado, la mayoría de los derechos, obligaciones y necesidades básicas de las personas, se expresan en la familia extensa de tres generaciones. Así, la abuela puede ser tan valiosa para su hija en el cuidado de los nietos/as, como la hija para su madre en caso de enfermedad o vejez. Esto da lugar a una reciprocidad de intereses, relaciones y servicios, en lo que algunos autores han venido a denominar "solidaridad intergeneracional" (Bazo, 1999; Adroher, 2000), concepto que depende a su vez de otros factores, entre los que se encuentran el estado de salud, la situación económica y la autonomía de los que conforman la familia.

En otro sentido, la imagen que dan de sí las personas de más edad es de una clara ruptura de la uniformidad que los colocaba a todos y a todas en un colectivo. Esa ruptura lleva consigo el levantamiento del "estigma" de la vejez como algo fatal, amargo y cerrado, y lleva a desarrollar distintas estrategias de adaptación que equivalen a otros tantos estilos de vida.

Los mayores no sólo se pliegan o se "adaptan" a diversas situaciones familiares o vitales, sino que ellos mismos ofrecen un tipo de relaciones que es apreciado por los niños. La mayor longevidad ha establecido un tramo más largo, más abierto a la propia planificación, a las propias expectativas del mayor de lo que sucedía en etapas pasadas. Lo cual nos lleva a pensar que las relaciones intergeneracionales se han modificado a lo largo del tiempo y, hoy por hoy, se entienden y se abordan desde perspectivas muy diferentes. Pasamos a exponer algunas de ellas.

\section{Experiencias innovadoras en el ámbito de la educación infantil. El caso de la asociación OFECUM (Oferta Cultural de Universitarios Mayores) en Granada (España)}

Como venimos diciendo, las relaciones intergeneracionales suponen una oportunidad única de vinculación entre distintos colectivos de la sociedad. No sólo sirven para compartir espacios de ocio y tiempo libre; sino intereses, ideas, sentimientos, apoyo... 
Estas relaciones pretenden una mayor integración, participación y transformación social a través de procesos de toma de conciencia personal y grupal. Potencian también una perspectiva crítica y reflexiva que posibilite la apertura hacia una sociedad de por sí compleja. Así se recoge en el Libro Blanco del Envejecimiento Activo de la Junta de Andalucía (España) (2010), donde se ha apostado por introducir la intergeneracionalidad como dimensión transversal:

\footnotetext{
"Siendo obvio que las diferencias entre unas personas y otras existen, necesitamos que esas diferencias no nos conduzcan a percibirnos como contrarios en un asunto que a todos nos interesa: vivir más tiempo pero, sobre todo, vivir mejor. La intergeneracionalidad nos plantea precisamente que el contacto, el intercambio y la solidaridad entre todas las generaciones, tienen resultados positivos a la hora de envejecer de forma activa" (Junta de Andalucía, 2010, pp. 347-348).
}

Ahora bien, para hablar de intergeneracionalidad no basta con "estar juntos", lo importante es "hacer y hacerse juntos", y que ese hacer vaya más allá de la mera interacción y pase a la relación. De esta idea surge uno de los posibles logros más relevantes de todo este esfuerzo en favor de la intergeneracionalidad:

\footnotetext{
“Quizá, mirando al horizonte del medio y largo plazo, habría que hablar de una sociedad para todas las generaciones, o de una sociedad intergeneracional, que facilite las relaciones entre las personas, unas relaciones naturales, provocadas por el deseo de estar juntos y hacer juntos sus respectivos recorridos existenciales" (Sánchez, Kaplan y Sáez, 2010, p. 44).
}

En la actualidad, los cauces de participación social entre las personas mayores pueden ser muy distintos. Uno de los que más énfasis está adquiriendo en los últimos años en España es el asociacionismo.

Si nos centramos en el asociacionismo como herramienta de participación social, destacamos las asociaciones de universitarios mayores; esto es, adultos mayores que están en relación directa con la universidad que les da cabida. Este tipo de asociaciones fomentan las actividades culturales y lúdicas y traba- jan constantemente por el reconocimiento social del colectivo que las integra.

En Granada (España), ciudad a la que pertenece nuestra universidad, podemos encontrar dos ejemplos de ellas: OFECUM (Oferta Cultural de Universitarios Mayores) y ALUMA (Asociación del Aula Permanente de Formación Abierta de la Universidad de Granada). Por experiencia y mayor conocimiento, vamos a centrarnos en OFECUM.

Esta asociación sin ánimo de lucro surge en 1998 a través de la iniciativa de universitarios mayores, con el objetivo de compartir experiencias a través de la cultura y cubrir una necesidad de intercomunicación. Pone al alcance de todos sus socios la información sociocultural producida en la ciudad de Granada, eliminando los obstáculos que impiden la recepción de la información o la asistencia a las actividades de todas las personas que lo desean.

OFECUM modificó el pasado año (2012) sus estatutos para transformar la asociación en "Intergeneracional", con el objetivo de que socios y voluntarios convivan en el desarrollo de las actividades en el afán de convertir la ciudad en una "sociedad para todas las edades". Así, el Presidente de esta entidad nombra su junta directiva, que pasa a estructurarse en vocalías, cuya responsabilidad se circunscribe a una serie de secciones las cuales cubren una o más áreas de actividad.

Los objetivos que esta asociación persigue son: a) Convertir nuestra comunidad en aula de cultura; b) Mejorar la calidad de vida de las personas mayores; c) Promover la solidaridad intergeneracional; d) Lograr la motivación ciudadana para la participación mediante la integración social; y e) Conseguir una plena autonomía de los mayores, para lo que pretende la integración de las personas a través de las nuevas tecnologías.

Para llegar a cumplirlos, OFECUM propone actividades y talleres dentro de la vocalía de proyectos, principalmente de perfil intergeneracional, entendiendo por tales la definición que ofrece "Generations United" (la entidad estadounidense que acoge en su seno a decenas de organizaciones dedicadas al trabajo intergeneracional), que utiliza el término "prácticas intergeneracionales" para referirse a aquellas: 


\begin{abstract}
"Actividades o programas que aumentan la cooperación, la interacción y el intercambio entre personas de distintas generaciones, y permiten a estas personas compartir sus talentos y recursos y apoyarse entre sí en relaciones beneficiosas tanto para las personas como para su comunidad"6.
\end{abstract}

Se llevan a cabo múltiples proyectos con varias generaciones desde hace más de una década, pero por la temática que recoge este artículo nos centraremos en los que conectan a los niños y niñas de Educación Infantil con las personas mayores. A continuación se presentarán.

\section{Proyecto "Cuánto cuentan los mayores": (2003-2005, Granada)}

Participan los siguientes centros educativos: Colegio Cristo de la Yedra, Colegio Público San José, Colegio Público Gómez Moreno, Guardería Infantil del Centro Penitenciario de Albolote/Granada y Asociación Granada Acoge.

Este proyecto trató de establecer una conexión entre tres elementos aparentemente sin relación: la tradición oral a través de los cuentos (adaptados a la edad), la intervención intergeneracional (los mayores cuentan cuentos en los tres centros escolares elegidos) y el envejecimiento productivo (los mayores contribuyen a resolver cuestiones realmente significativas para todos, de forma eficaz para la mejora de las actitudes hacia el envejecimiento y la eliminación de estereotipos acerca de las personas mayores).

Se pretendió que, después del período de formación como cuentacuentos de los mayores y sus "contadas" realizadas, los niños de estos centros escolares entrasen en un contacto periódico con estas personas mayores, para llevar a cabo actividades sostenidas en el tiempo, que permitiesen establecer lazos sociales duraderos de amistad y afecto con los que mejorar las actitudes y los comportamientos hacia los mayores.

\section{Proyecto "Uniendo generaciones = rompiendo barreras"}

6 Para mayor información al respecto puede consultarse la página web de la asociación, http://www.ofecum.es/
Intervención de los mayores en el Aula de la Guardería -niños y padres internos conjuntamente- del Centro Penitenciario de Albolote (Granada) (2005-2009).

Es fácil imaginar con exactitud la situación de aislamiento y exclusión social de estos padres, madres e hijos. Además, en muchos casos, los niños carecen de contacto con personas mayores y su mundo afectivo y relacional se restringe al conformado por sus padres, aunque en ocasiones ni siquiera tienen contacto con ambos, sino únicamente con la madre.

Los funcionarios de la prisión y las educadoras infantiles que se ocupan de ellos son su tabla de salvación, y eso es lo que el programa, entre otras cosas, les facilitaría: contacto periódico y actividades con personas mayores que utilizarían los cuentos, entre otras herramientas (canciones, disfraces, meriendas, juegos didácticos, manualidades, etc.), para ofrecer afecto $\mathrm{y}$ apoyo social a esas madres, padres y niños/niñas.

\section{Proyecto "GYRO: Generativa y relevancia social de personas mayores"}

Desarrollado en: Colegio Cristo de la Yedra, Colegio Público San José, Colegio Público Gómez Moreno y en el Aula de la Guardería del Centro Penitenciario de Albolote/Granada (Granada. 2005 a 2006, subvencionado por La Obra Social Caja Madrid).

Este proyecto trató de mejorar la relevancia social, la participación y el bienestar de las personas mayores residentes en tres de los más envejecidos distritos de la ciudad de Granada (Albaicín, Beiro y Centro). Para ello, utiliza los programas intergeneracionales que fomentan las oportunidades de relación entre mayores y centros educativos de los distritos en los que residen, para aumentar la relevancia social de los mayores a través de la puesta en marcha de actividades con niños, buscando lograr un incremento de la generatividad de los mayores $\mathrm{y}$, por ende, de su bienestar.

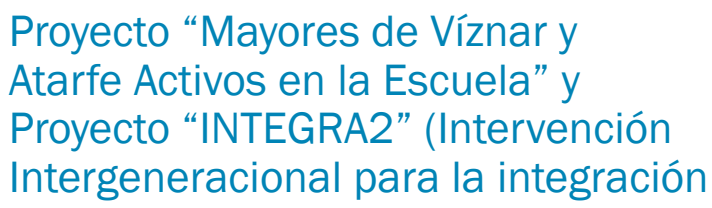




\section{No se pretende simplemente organizar actos donde personas mayores cuenten cuentos a niños españoles e inmigrantes, sino ir más allá, fomentando un aprendizaje significativo para cada una de las partes"}

\section{social de hijos de familias inmigrantes residentes en 2 municipios de Granada)}

Desarrollado en los Municipios de Atarfe y Víznar de Granada, en 5 colegios de la provincia. Estos programas han tratado de establecer una conexión, en la práctica, entre tres elementos: los cuentos, la intervención intergeneracional y la acogida e integración social de las personas inmigrantes. No se pretende simplemente organizar actos donde personas mayores cuenten cuentos a niños españoles e inmigrantes, sino ir más allá, fomentando un aprendizaje significativo para cada una de las partes.

El objetivo ha sido ambicioso: utilizar el intercambio de cuentos de las culturas de la población autóctona e inmigrante como gesto de valoración y acogida intercultural. Todo ello en clave intergeneracional, es decir, con la participación de mayores cuentacuentos autóctonos y de niños/as españoles e inmigrantes, dado que la intervención intergeneracional ha demostrado ya su idoneidad para el trabajo de acogida a inmigrantes.

\section{Proyecto "MENTOR: por cada mayor MENtor un niño inmigrante lecTOR" (fase 1 y fase 2 )}

Colegio Público San José en horario extraescolar. Granada. Fase 1 -Enero 2006/Marzo 2006- y fase 2 -Septiembre 2006/ Junio 2007-. (Fase 1 subvencionada por la Junta de Andalucía y coordinado externamente).

El proyecto pretende mejorar el acceso y el desarrollo de la educación entre los grupos más desfavorecidos, erradicando el analfabetismo y el absentismo escolar y favoreciendo la escolarización e integración escolar. Así mismo, trabaja en el fomento de actitudes sociales de solidaridad, tolerancia activa e igualdad de trato hacia las familias inmigrantes.
El objetivo es favorecer la convivencia en un clima social de respeto y tolerancia a la pluralidad, para lo que se fomenta la inclusión de menores inmigrantes en actividades que supongan una colaboración, diálogo, solidaridad y no discriminación, bajo el lema: "Los valores no se enseñan, se contagian".

\section{Proyecto "Mayores activos en la escuela"}

Colegios de Granada (Cristo de la Yedra, Colegio Público San José, Colegio Público Gómez Moreno y Escuela Infantil del Centro Penitenciario de Albolote/Granada); Colegios de la Provincia (Colegios del Municipio de Víznar (Colegio Arzobispo Moscoso y Escuela de Adultos), Colegios del Municipio de Atarfe (Colegio Atalaya, Colegio Doctor Jiménez Rueda, Colegio Sagrada Familia y Escuela de Adultos).

En las fases 1 y 2 (2007-2008), se pretendió una mejora de la calidad de vida de las personas mayores mediante su implicación como voluntarios (significativos) en programas intergeneracionales (PIs.). Esa mejora sólo sería posible promoviendo el envejecimiento activo a través de la participación (activa) de los mayores en su entorno; en concreto, el proyecto les dio la oportunidad de participar y de contribuir, con su experiencia y recursos, en la realización de actividades en beneficio para niños de centros escolares de Granada y la Guardería del Centro Penitenciario de Granada -niños conjuntamente con su padres, internos de la prisión-.

Las fases 3 y 4 se llevaron a cabo en los colegios: Cristo de la Yedra, Colegio Público San José y Guardería del Centro Penitenciario de Albolote/Granada, del 2008 al 2009 y 2009 a 2010 y fueron subvencionadas por OFECUM. En esta tercera y cuarta fase se pretendió afianzar la presencia de los mayores en los lugares indicados.

En la quinta fase, se incorporó al Colegio José Hurtado (Noviembre 2010 hasta Abril de 2011). Este proyecto aprovecha el aula en horario lectivo como espacio común en el que mayores 


\section{OFECUM tiene claro la importancia de trabajar en red. Debemos transmitir a las instituciones públicas de la comunidad y su entorno la importancia de compartir recursos y experiencias"}

y niños pueden seguir reuniéndose para potenciar el envejecimiento activo mediante el voluntariado significativo, mejorar las oportunidades de enseñanza y aprendizaje tanto para los mayores como para los niños (proyecto intergeneracional) y rescatar la tradición oral.

Se desarrollaron talleres de cuentos en valores, expresión corporal, coro intergeneracional, montaje de obras de teatro, relajación a través del cuento, educación para la ciudadanía, literatura artística, jardinería y rehabilitación de un huerto, y educación emocional.

\section{Proyecto: "Red intergeneracional de Granada y la Provincia -INTERGEGRA- Granada"}

Desarrollado desde julio 2006 hasta julio 2008. Adheridos oficialmente a la Red mediante la firma del "acuerdo de adhesión" a la misma: Asociación Anaquerando; Ayuntamiento de Pulianas (área de cultura); ASPACE Granada (Ssociación de Parálisis Cerebral); ONG Solidarios para el Desarrollo; ALUMA (Asociación de Alumnos Universitarios del Aula de Mayores de la Universidad de Granada); y OFECUM.

OFECUM tiene claro la importancia de trabajar en red. Debemos transmitir a las instituciones públicas de la comunidad y su entorno la importancia de compartir recursos y experiencias ya existentes de los proyectos que, desde OFECUM y otros entornos -incluso internacionales-, se han ido cosechando en el campo intergeneracional, para exportarlos a la provincia a través de una Red de Proyectos Intergeneracionales que rescaten la intervención intergeneracional en la zona de Granada. Con ello se intenta demostrar: “'Cuánto cuentan los mayores de la provincia de Granada!”. En junio de 2006, OFECUM lideró la creación de la Red Intergegra, que pretende interrelacionar mayores y jóvenes para el beneficio de ambos. Actualmente, las reuniones de la Red se mantienen suspendidas para la reflexión de cada entidad.

\section{Proyecto "Una escuela infantil para todas las edades"}

Realizado en la E.I.M (Escuela Infantil Municipal) Belén, la E.I.M Luna, y la E.I.M Duende, gestionadas por OFECUM y la Fundación Granada Educa (Concejalía de Educación del Ayuntamiento de Granada); es un proyecto subvencionado por la Fundación y OFECUM Granada. Se desarrolló en la ciudad entre los períodos de Noviembre de 2009- Mayo de 2009, y Noviembre 2010-Mayo 2010.

El proyecto se planteó en varias fases: la fase 1 y 2 , para introducir y potenciar las buenas relaciones intergeneracionales como pieza clave en el proyecto educativo de las Escuelas Infantiles del Ayuntamiento de Granada. Con ello se pretendió aprovechar la presencia cotidiana, programada, continuada y significativa de personas de distintas generaciones en la escuela infantil, para ir haciendo del espacio escolar una comunidad de diversas generaciones en la que se aprecie y valore la contribución de todos y cada uno de sus miembros, con independencia de la edad que tengan o de la generación a la que pertenezcan.

La tercera fase se desarrolló entre Noviembre de 2010 y Mayo de 2011 e introdujo en tres de las Escuelas de la Fundación, algunas personas mayores que, en calidad de voluntarias, acudían de forma periódica y programada a desempeñar tareas educativas significativas previamente acordadas. Estas tareas estaban enmarcadas en el contexto educativo de las escuelas, a través de varios talleres de trabajo intergeneracionales que se desarrollaron semanalmente en sesiones de $1 \mathrm{~h}$ y/o $2 \mathrm{~h}$ : Taller de Cocina, Taller de Invernadero y Taller de Bordado.

Este proyecto supone un acercamiento considerable a la creación de una verdadera sociedad para todas las edades, puesto que los implicados son muy diversos y el enriquecimiento es observable. 


\section{Proyecto Intergeneracional: “Educando todos juntos"}

Realizado en la E.I.M (Escuela Infantil Municipal) Belén, la E.I.M Duende, la E.I.M Juan Latino, el Colegio PP Escolapios y el Colegio Ave $\mathrm{M}^{\mathrm{a}}$ San Cristóbal, gestionadas por OFECUM y la Fundación Granada Educa (Concejalía de Educación del Ayuntamiento de Granada). Proyecto subvencionado por la Fundación y OFECUM en Granada, de Febrero 2012 a Diciembre de 2012.

Es el proyecto más reciente. Pretende integrar a los mayores en la vida de la escuela de manera estable y estructural, siempre con la idea de que su integración debe estar sometida a los criterios de espacio y tiempo que previamente se acuerden para cada propuesta concreta. Se realiza a través de talleres que dirige el voluntario mayor y que benefician a toda la comunidad educativa, puesto que todos se ven implicados en ese proceso de crecimiento como personas, en el respeto, la tolerancia y la creación de una sociedad para todos, sin discriminación.

Cabe aclarar que los proyectos intergeneracionales que ha estado desarrollando OFECUM en Granada, desde el 2003 hasta la actualidad, han contado con el apoyo financiero y/o con la colaboración en diversas facetas, de instituciones, entidades y voluntariado. Además, OFECUM cuenta con 350 socios y en estos programas habrán participado alrededor de 300 voluntarios.

Tras la realización de cada actividad dentro de los proyectos, los voluntarios realizan una ficha de evaluación que recoge sus impresiones, positivas y negativas, además de anécdotas u observaciones relevantes acerca de la puesta en marcha del taller.

Los niños y niñas hacen una evaluación mucho más personal, puesto que no llenan ningún tipo de ficha, pero sí se aprecia un cambio considerable en ellos, observado por maestros y familiares. Quizás sería bueno para la asociación contar con una evaluación más estructurada para los alumnos de las escuelas infantiles y colegios, distinta de los aportes que hacen los tutores de clase sobre cómo creen que ésta ha influido en sus alumnos, y que se recoge en la memoria final del proyecto.

Los voluntarios mayores, antes de realizar su participación en los centros educativos, reciben una formación para abordar su labor con la confianza de ser un buen cuentacuentos o saber dirigir una sesión de relajación/meditación, a través de una historia con moraleja o un cuento.

Todo ello se realiza bajo el lema "Sembrando Valores", puesto que lo fundamental en estas relaciones intergeneracionales, para OFECUM, es transmitir valores que, consideran, se están perdiendo en la sociedad actual: solidaridad, compañerismo, amistad, tolerancia, respeto, creatividad, justicia, sinceridad, nobleza, paz interior, etc.

En concreto, las sesiones de relajación son una vía de escape incluso para los más pequeños, que ya son protagonistas de un mundo agitado, y comienzan con una breve presentación al grupo clase, seguida de unas instrucciones básicas ("Vamos a relajarnos, cerramos los ojos, estamos en silencio, etc.").

Tras esta primera parte, comienza la respiración pulmonar dirigida, que durará unos 3-5 minutos, dependiendo de la edad y estado de los alumnos ("Entra el aire por la nariz y lo soltamos por la boca, vamos a ponerle un color claro al aire que entra y un color oscuro al que sale..."). Posteriormente, se dirige la relajación muscular, de abajo a arriba, comenzando por los dedos de los pies, hasta llegar al cuero cabelludo. A continuación, se lee un cuento adaptado a la edad y al mensaje que se quiera trasmitir, lo que dura unos 2 minutos.

Poco a poco, se les dan instrucciones para que vayan moviendo su cuerpo y abran los ojos. Finalmente, se despide la sesión con una rueda de valoración, con la salvedad de que no se puede decir ni bien, ni mal. Estas sesiones suponen experiencias irrepetibles de las que, tanto mayores como niños, salen entusiasmados. Valga esta anécdota como ejemplo:

Luis, un niño de 5 años de una escuela infantil granadina, iba por la calle con su padre cuando se encontró con José Antonio, el director de la actividad, e inmediatamente se lanzó a sus piernas. Su padre, extrañado, le preguntó: “¿Y este señor quién es?" A lo que Luis respondió: "Este es... el de la tranquilidad".

También se llevan a cabo sesiones de cuentacuentos, con niños de Educación Infantil y de Educación Primaria, que finalizan con un dibujo, realizado por los niños y niñas de la cla- 

aprendizajes y vivencias que se dan dentro del aula a través de la sabiduría y el cariño de las personas mayores"

se, que funciona como evaluación de lo que para los alumnos resultó más significativo del cuento. Esto es sólo una mínima parte de lo que las personas mayores pueden hacer por la sociedad en general y, concretamente, por la infancia. La gran labor que llevan a cabo ha de ser difundida y experimentada en nuestra propia vida para así apreciar que la sociedad puede cambiar, indiscutiblemente, para bien.

\section{Conclusiones}

Tal como exponíamos al principio de este artículo, la educación infantil en España constituye, hoy por hoy, una etapa con entidad propia que persigue ante todo el desarrollo de valores democráticos, teniendo en cuenta la diversidad cultural, la cultura de paz, los hábitos de consumo y vida saludable, la igualdad y el respeto de los derechos y libertades fundamentales, entre otros muchos.

Esto conlleva la necesidad de proponer estrategias y metodologías concretas que, desde un trabajo coordinado por los propios profesionales, pero con la implicación y participación activa de toda la comunidad educativa, permita el aprendizaje mutuo desde formas diversas de comunicación y diálogo con el entorno próximo. A través de las experiencias innovadoras que hemos expuesto y que parten de las relaciones intergeneracionales como fundamentación y guía, se ha logrado una mayor asimilación por parte de todos los participantes, de los valores cívicos y democráticos que se perseguían.

Pero, además, su desarrollo ha permitido un mayor acercamiento entre colectivos de edades diversas, rompiendo estereotipos y barreras que provienen más del desconocimiento que de la propia realidad. Para las personas mayores supone un mayor protagonismo en la sociedad actual, al posibilitarles demostrar que son capaces de acercarse a la infancia desde la experiencia y la fortaleza que devienen del tiempo vivido. Esto les hace "rejuvenecerse" en cuerpo y espíritu y dota de mayor significación sus relaciones con los más pequeños.

Para los niños, es esencial que encuentren cercanos y relevantes los aprendizajes y vivencias que se dan dentro del aula a través de la sabiduría y el cariño de las personas mayores, a quienes sienten como agentes transmisores de valores y rescatadores de una cultura que, sin ellos, quedaría posiblemente perdida en el tiempo.

Como educadores, nuestro papel es decisivo, actuando como mediadores y facilitadores de estrategias que permitan, a su vez, poner en marcha los mecanismos adecuados, dirigidos hacia una optimización de los procesos intergeneracionales. Teniendo como referente el carácter global y permanente de la educación, el fomento de la participación a este respecto habrá de tener en cuenta las características evolutivas que definen determinados comportamientos, los elementos socio-históricos y, en suma, los condicionantes personales intervinientes.

Todos aprendemos de todos en niveles distintos, pero la finalidad última es el desarrollo de competencias, por parte de adultos y pequeños, que nos permitan comprender y mejorar nuestras relaciones $\mathrm{y}$, en definitiva, nuestra participación $\mathrm{y}$ compromiso con la sociedad de hoy.

Quisiéramos agradecer de forma expresa la colaboración desinteresada de la Asociación OFECUM, que nos facilitó toda la información precisa para realizar este artículo. Sirva éste de reconocimiento y admiración por el trabajo encomiable que desarrollan, confiando en que suponga de igual modo un nuevo impulso para seguir generando procesos de encuentro e implicación entre distintas generaciones. 


\section{Referencias}

Adroher, S. (Coord.). (2000). Mayores y Familia. Madrid: Universidad Pontificia Comillas e IMSERSO.

Bazo, Ma T. (1990). La sociedad anciana. Madrid: Centro de Investigaciones Sociológicas y Siglo XXI de España Editores S.A.

Bazo, Mª T. (Coord.). (1999). Envejecimiento y sociedad: Una perspectiva internacional. Madrid: Editorial Médica Panamericana.

García Mínguez, J. y Bedmar Moreno, M. (2002). Hacia la Educación Intergeneracional. Madrid: Dykinson.

Junta de Andalucía (2010). Libro Blanco del Envejecimiento Activo. Sevilla: Consejería para la Igualdad y Bienestar Social.

Montero, I. (2003). "La educación intergeneracional: ¿Utopía o realidad?”. En M. Bedmar e I. Montero (Coords.). La educación intergeneracional: un nuevo ámbito educativo. Madrid: Dykinson, 111-124.

Montero, I., Bedmar, M. (2009). "Reconstrucción de vivencias desde la educación intergeneracional: experiencia docente e innovación a través de la historia oral". En VII Congreso Internacional de Historia Oral. México: Universidad de Colima.
Montero, I., García Mínguez, J. y Bedmar, M. (2011). "Ciudadanía activa y personas mayores. Contribuciones desde un modelo de educación expresiva". Revista Iberoamericana de Educación. RIE $n^{\circ}$ 55/5. Obtenido el 20 de Enero de 2013, desde http://www.rieoei. org/deloslectores/3934Montero.pdf

Rochat, P. (2012). "La autoconciencia y la "conciencia moral" en el desarrollo". Revista Infancia y Aprendizaje, 35 (4), 387-404.

Sáez, J. (2002). Pedagogía social y programas intergeneracionales: Educación de personas mayores. Málaga: Ediciones Aljibe.

Sánchez, M., Kaplan, M. y Sáez, J. (2010). Programas intergeneracionales. Guía Introductoria. Madrid: IMSERSO.

Santamaría, C. y Marinas, M. (2009). "Percepción en niños y adolescentes de las personas mayores". En Perfiles y Tendencias, Boletín sobre el Envejecimiento $N^{o} 38$. Madrid: IMSERSO.

UNICEF. (2012). La infancia en España 2012-2013. El impacto de la crisis en los niños. Madrid: UNICEF. 


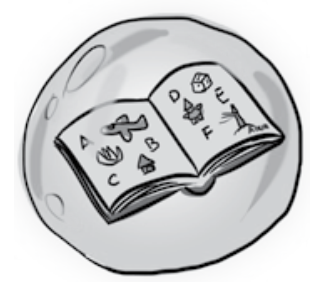

